\title{
Brücke zwischen Spezialisten und Grundversorgern
}

Wer einen renommierten medizinischen Preis erhält, ist in aller Regel in der Forschung tätig und auf seinem Gebiet so spezialisiert, dass die allgemeinverständliche Vermittlung der auszeichnungsträchtigen Erkenntnisse kein einfaches Unterfangen ist. Der Hans-Debrunner-Preis hat eine andere Stossrichtung: Er stellt einen Versuch dar, eine Brücke zwischen Spezialisten - in diesem Fall den Orthopäden - und den medizinischen Grundversorgern zu schlagen.

Bruno Kesseli
Medizinische Forschung bleibt unfruchtbar, wenn ihre Resultate nicht Eingang in die Praxis finden und somit den Patienten zugute kommen. Dieser an sich selbstverständliche, aber zentrale Gedanke liegt dem Hans-Debrunner-Preis zugrunde, der im letzten Jahr erstmals verliehen wurde. Nicht die «einsame» Arbeit an der Spitze wird ausgezeichnet, sondern der Wissenstransfer von der Spitze an die Basis. Ein Ansatz, der bisher eher exotischen Charakter hat, der aber angesichts der schwierigen Perspektiven der medizinischen Grundversorgung durchaus als verdienstvoll eingestuft werden darf.

\section{Spezialisiertes Wissen für die Praxis nutzbar machen}

Der Name des Preises, der unter dem Patronat der Schweizerischen Gesellschaften für Orthopädie (SGO) und Allgemeinmedizin (SGAM) steht, geht auf Hans Debrunner (1889-1974) zurück. Als Professor und Ordinarius für Orthopädie an der Universität Basel hat er sich gemäss seinem Sohn, Alfred M. Debrunner, ein Leben lang dafür eingesetzt, dass die unübersehbare Menge von Forschungsresultaten gesichtet, evaluiert, auf Nachhaltigkeit geprüft, konzentriert, zusammengefasst und in eine verständliche Form gegossen,

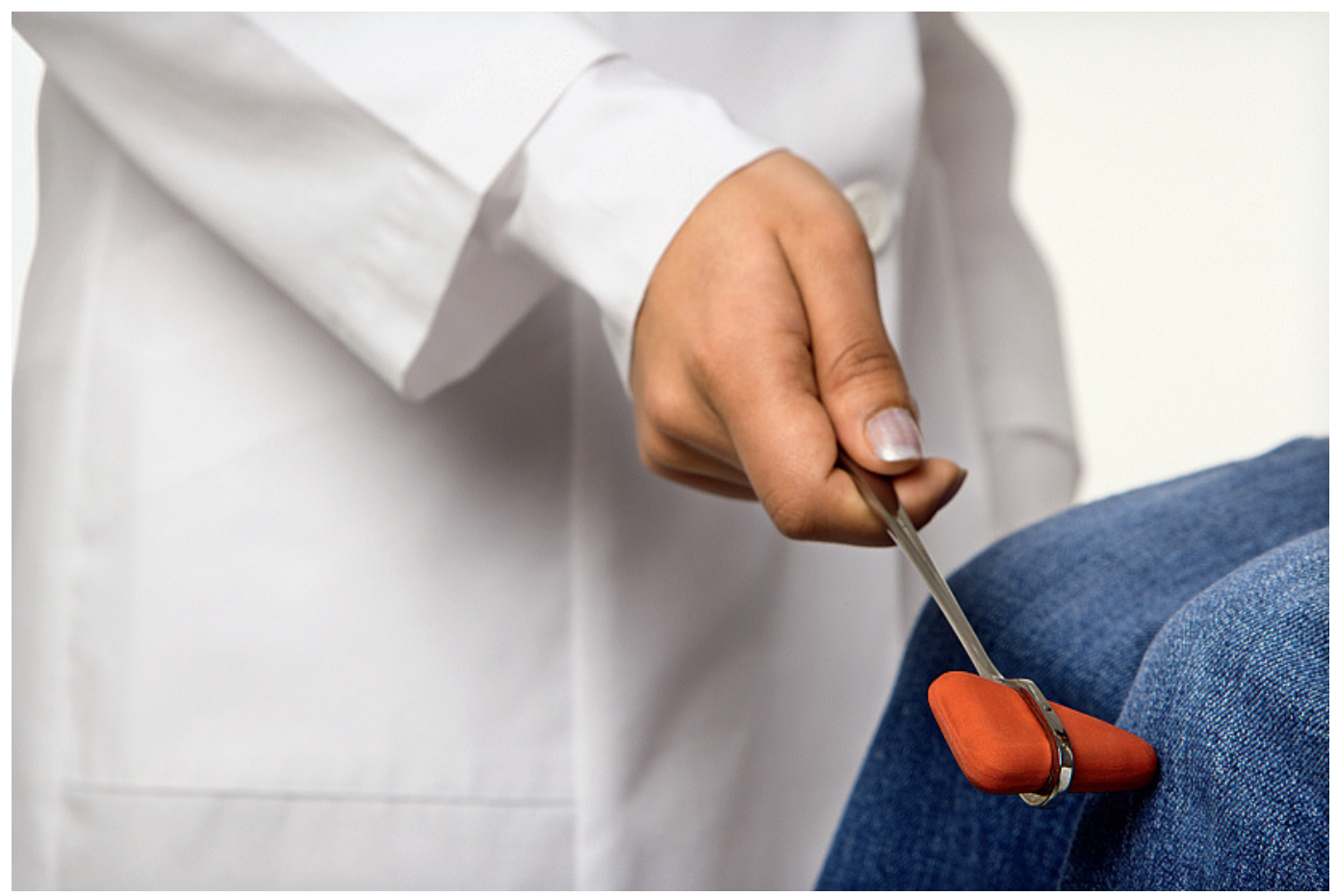

Mehr als ein defekter Apparat: Hinter dem Hans-Debrunner-Preis steht eine Auffassung von Medizin, die den Menschen als Individuum in den Mittelpunkt stellt. 
kurz: für die Aus-, Weiter- und Fortbildung und somit für die Praxis brauchbar gemacht wird.

Diesem Anliegen seines Vaters fühlt sich auch Alfred M. Debrunner - ebenfalls Orthopäde -, der den Preis ins Leben gerufen hat, verpflichtet. Aus seiner Sicht ist allen gegenteiligen Beteuerungen zum Trotz die Gefahr gross, dass der Mensch angesichts der enormen technischen Möglichkeiten der aktuellen Medizin als eine Art defekter Apparat gesehen und behandelt wird. Sein Leitbild - und das seines Vaters - wäre dagegen eine Orthopädie bzw. eine Medizin mit «menschlichem Antlitz», ein «menschenfreundliches Arzttum, das die Patienten als Individuen in den Mittelpunkt stellt».

\section{Schlüsselfunktion der Sprache}

Die Sprache als Mittel der Verständigung spielt im Zusammenhang mit dem Hans-DebrunnerPreis eine wesentliche Rolle. Genauso wie das

Ausschreibung Hans-Debrunner-Preis 2009 Der Hans-Debrunner-Preis bezweckt die Förderung der Zusammenarbeit zwischen Orthopäden und Hausärzten. Es handelt sich um einen Förderpreis zu Ehren von Hans Debrunner, der 1948-1959 als Professor und Ordinarius für Orthopädie an der Universität Basel wirkte. Der unter dem Patronat der Schweizerischen Gesellschaften für Orthopädie (SGO) und Allgemeinmedizin (SGAM) stehende Preis geht auf die Initiative von Hans Debrunners Sohn Alfred zurück, der ebenfalls Orthopäde ist. Der Preis wird für Arbeiten vergeben, die eine Brücke zwischen Spezialisten (insb. Orthopäden) und Hausärzten schlagen. (2008 ging ein Anerkennungspreis an einen Hausarzt).

Ausgezeichnet werden Lehrbücher, Publikationen, IT-Technologien und Veranstaltungen in Aus-, Weiter- und Fortbildungen, die in einfacher, klar verständlicher Form und Sprache den Hausärztinnen und Hausärzten, den Spe-
Arzt-Patienten-Gespräch in einer verständlichen Weise geführt werden soll, kommt der Sprache im Hinblick auf die Nutzbarmachung von hochspezialisiertem Wissen für die Praxis eine Schlüsselfunktion zu.

Vor diesem Hintergrund ist es naheliegend, dass der Preis vor allem für Arbeiten vergeben wird, die dem Nichtspezialisten in verständlicher, aber auch eleganter Sprache orthopädisches Grundlagenwissen vermitteln, das ihm für die praktische Arbeit mit seinen Patienten von Nutzen ist. Dabei kann es sich um Lehrbücher, Publikationen oder nachhaltige Veranstaltungen in Lehre sowie Weiter- und Fortbildung handeln, die Ärztinnen und Ärzten helfen, ihre Patienten bei Krankheiten und Unfallfolgen am Bewegungsapparat besser zu beraten, zu betreuen und zu behandeln. Die Zusammensetzung der Jury, in der Orthopäden und Grundversorger vertreten sind, trägt diesen Kriterien Rechnung.

zialärzten oder Studierenden die Grundlagen des Spezialfaches Orthopädie vermitteln und ihnen Hilfe für die praktische Arbeit mit ihren Patienten geben. Einreichung der Arbeiten elektronisch und auf Papier in 2 Exemplaren. Die Preissumme beträgt insgesamt 25000 Franken. Sie kann für eine oder mehrere Arbeiten vergeben werden.

Die Jury besteht aus Vertretern der SGO, der Hausarztmedizin und weiteren Fachpersonen. Sie wird präsidiert durch Prof. Dr. med. Peter Tschudi, Institut für Hausarztmedizin, Universität Basel.

Einreichungsschluss ist der 11. April 2009

Die Preisverleihung findet am 25. Juni 2009 anlässlich des SGO-Jahreskongresses in Genf statt.

Preisbüro und Korrespondenz: Schneiter \& Partner AG, Buchholzstrasse 122, Postfach, 8053 Zürich, Tel. 04499180 80, E-Mail: schneiter.komm@bluewin.ch 